\title{
Effects of fishing on reef fish communities at Pedro Bank and Port Royal Cays, Jamaica
}

\author{
J. Anthony Koslow ${ }^{1,2}$, Fred Hanley ${ }^{2}$, Robert Wicklund ${ }^{3}$ \\ ${ }^{1}$ Oceanography Department, Dalhousie University, Halifax, Nova Scotia, Canada B3H 4J1 \\ ${ }^{2}$ Zoology Department, University of the West Indies, Kingston, Jamaica \\ ${ }^{3}$ Caribbean Marine Research Center, Lee Stocking Island, Bahamas
}

\begin{abstract}
We examined the results of a natural 'experiment' to determine the effects of fishing on Caribbean reef fish communities. We repeated a trap survey in 1986 of reef fish populations at exposed, offshore sites on southeast (SE) and southwest (SW) Pedro Bank and protected, nearshore sites around the Port Royal Cays (PRC), Jamaica, that had been surveyed in 1969-73. The 'control' area - SW Pedro Bank - was virtually unexploited in 1969-73 and was lightly-to-moderately exploited in 1986. As 'treatment' areas, exploitation of SE Pedro Bank increased from moderate to heavy between surveys, and from heavy to very heavy around PRC. Overall catch rates declined significantly in heavily exploited areas - $82 \%$ over SE Pedro Bank and $33 \%$ around PRC - but did not decline over SW Pedro Bank. The largest fishes commonly caught in the traps (lutjanids and large serranids and scarids), tamilies particularly vulnerable to trap fishing (acanthurids and balistids), and other commercially preferred species groups (small serranids, haemulids, mullids) declined most consistently. Increases in catch rate were generally found only in commercially less desirable reef fishes, such as chaetodontids, holocentrids, and tetraodontiformes. The catch (i.e. species) composition changed significantly between surveys in heavily exploited areas (SE Pedro Bank and PRC) but not over SW Pedro Bank. Similar changes in 2 different reef environments (SE Pedro Bank and PRC) suggest that quantitative relationships of catch rate and composition with fishing effort may be useful in fishery management.
\end{abstract}

\section{INTRODUCTION}

Reef fish comprise a major fish resource in the Caribbean and form the basis of artisanal fisheries throughout the region. These fisheries are virtually unmanaged outside the USA and its territories, and little is known of their impact upon the fish communities, except that overall catch rates decline in heavily exploited areas (Munro 1983 p. 10-14, Caribbean Fishery Management Council 1985). The reef habitat associated with many Caribbean islands is limited, and the home range of many fishes associated with the reefs is highly restricted. Although fish traps set from open 'canoes' are the primary gear, entire reef communities are subject to unregulated exploitation, which appears to be generally increasing due to conversion to motorized 'canoes', expanding human population and lack of alternative employment in chronically depressed economies (Munro 1983, Caribbean Fishery Management Council 1985). Large-scale change of reef fish communities may affect the entire reef system, as well as the fisheries themselves, because of close links

(c) Inter-Research/Printed in F. R. Germany between fish, algal, and coral dynamics (Neudecker 1979, Hay 1984).

We examine here the results of a natural experiment' to study the impact of fishing on reef fish communities. From 1969-73, reef fish populations around the Port Royal Cays (PRC) outside Kingston, Jamaica (pop. ca 700 000), and on Pedro Bank were intensively surveyed (Munro \& Thompson 1983) (Fig. 1). These areas experienced widely varying histories of fishing both before and after this survey (Table 1). PRC had been exploited for at least 140 yr (Gosse 1851), was heavily exploited in 1968, and is more intensely exploited today, although landings have not increased since 1968. On the other hand, significant exploitation of Pedro Bank only began in the 1960s and has always been concentrated in the southeast (SE) portions of the bank, where cays serve as bases for fishermen. SE Pedro Bank thus serves as a 'treatment' area. It was only lightly-to-moderately fished in $1969-73$ but is today very intensively fished, with some 500 fishermen living on the cays and fishing from motorized 'canoes' in their environs. However, the remote southwest (SW) 

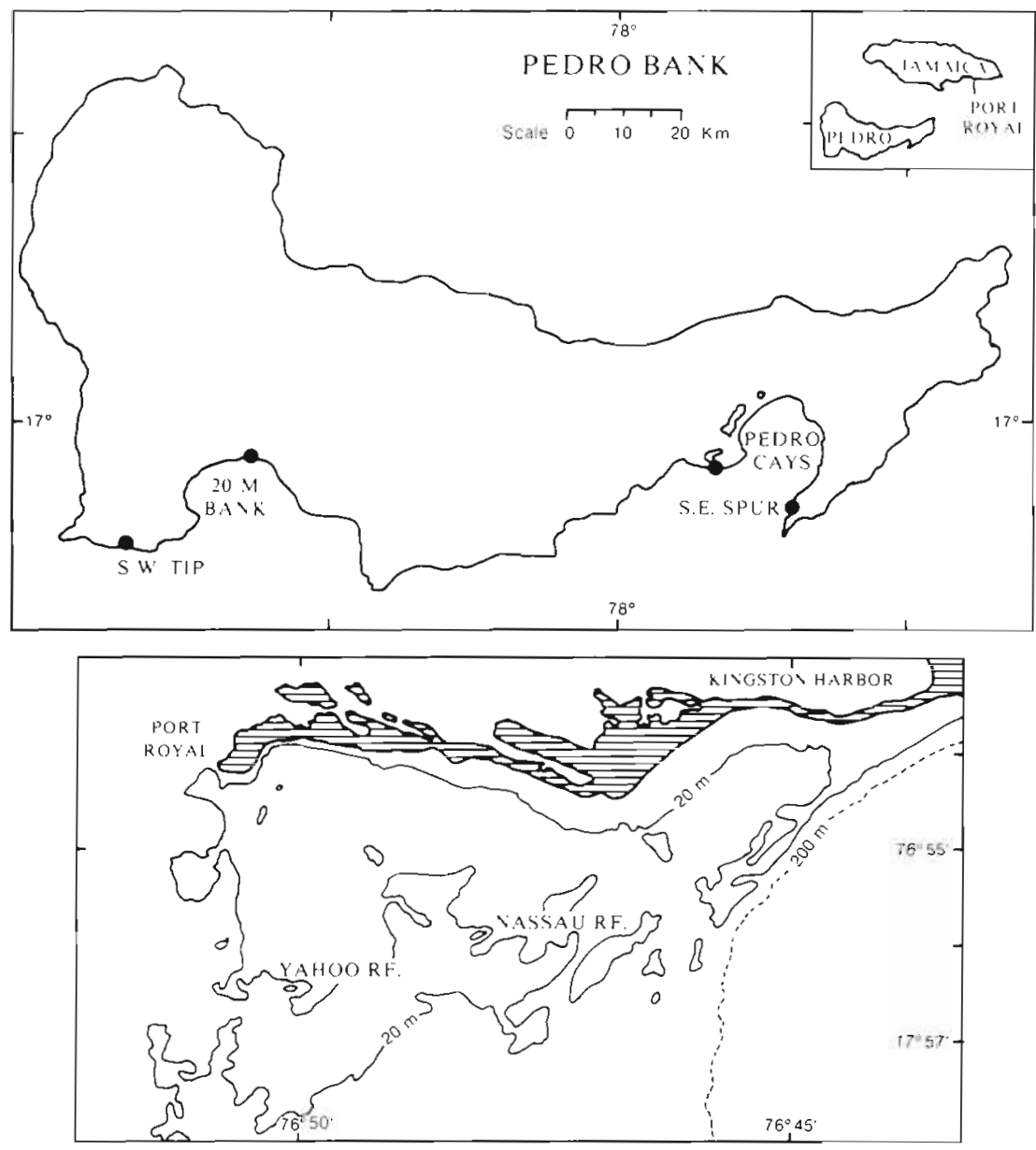

Fig. 1. Port Royal Cays area and Pedro Bank showing trap survey sites. The depth contour shown for Pedro Bank is the $200 \mathrm{~m}$ isobath, which generally overlies the $50 \mathrm{~m}$ isobath

portion of Pedro Bank, lacking any base for the fishermen, serves as a 'control'. It was virtually unexploited in 1969-73 and in 1986 was still only moderately fished (Table 1).

SE and SW Pedro Bank share a similar oceanic environment. Reef habitat is found predominantly along the edge of the bank, particularly in the southwest (Dolan 1972) and is composed of a generally hard pavement with low coral relief at 20 to $35 \mathrm{~m}$ depth. The bank is remote from human influence except for fish- ing. We therefore expected to find broadly similar fish communities on the 2 sides of the bank in 1969-73, with the greatest changes in the intervening 15 yr over the heavily exploited SE portion of the bank.

The sampling sites around PRC represent a markedly different reef environment: large patch reefs extending from near-surface to $20 \mathrm{~m}$ within protected waters behind a fringing reef. The PRC have not been significantly affected by hurricanes in recent decades, unlike the north coast of Jamaica. They are influenced by

Table 1. Fishing effort (numbers of canoes) and reef fish landings in metric tons from the south Jamaica shelf, which includes Port Royal Cays and Pedro Bank from the periods 1968-74 and 1981-86. Data sources: 1, Munro 1983; 2, Sahney 1982; 3, M. Haughton, Fisheries Div., Kingston. Jamaica, pers comm.; 4, spot surveys by authors of fish trap density at sampling sites

\begin{tabular}{|lcccc} 
& \multicolumn{2}{c}{ Fishing effort (canoes) } & Landings (t) \\
& $1968-74$ & $1981-86$ & $1968-74$ & $1981-86$ \\
\hline S. Jamaica Shelf (total) & (motorized) & $1079^{1}$ & $1400(+30 \%)^{2}$ & $3500^{2}$ \\
SE Pedro Bank & $658^{1}$ & $1183(+80 \%)^{2}$ & $3500^{1}$ \\
SW Pedro Bank & $72^{1}$ & $150-200(+108-178 \%)^{3}$ & \\
Pedro Bank (total) & $\sim 0^{1}$ & $10 \%$ of SE Pedro & $756^{1}$ & $1600^{2}$ \\
\hline
\end{tabular}


outflow from Kingston Harbour, but the discharge flows predominantly to the west (i.e. away from PRC). There are considerable differences in the composition of the fish communities at PRC and Pedro Bank (Munro 1983). Comparison of changes in the fish communities of PRC and SE Pedro Bank should indicate how fishing affects fish communities in different reef environments.

What changes might one expect to encounter due to the influence of trap fishing? Little is known about the selectivity of fish traps. If they are unselective within a given size range, all fish groups within that size range might be expected to decline at comparable rates. However, although traps catch a broad spectrum of the reef community, piscivores (e.g. serranids and lutjanids) seem particularly attracted to fish traps by the bait as well as by the prey fish, which appear to enter the trap for cover. One might also expect large fish (e.g. large serranids, lutjanids, and scarids) and deepbodied fish (e.g. pomacanthids and balistids) to decline disproportionately because they are retained by the traps at an earlier age relative to their age of maturity. High rates of exploitation also generally tend to favor smaller species with higher natural rates of increase.

\section{METHODS}

Our 1986 survey followed the methods used in the 1969-73 survey (Munro \& Thompson 1983). We selected sites previously surveyed around PRC and on SE and SW Pedro Bank (Fig. 1) and used the gears primarily employed in that survey: unbaited wooden Antillean Z-traps around PRC, together with lightlybaited stackable metal traps on Pedro Bank. Trap meshes were standard $3.2 \mathrm{~cm}$ (1.25 in) chicken wire. Our traps soaked for 1 to $5 \mathrm{~d}$ at PRC sites and for $1 \mathrm{~d}$ on Pedro Bank. All fish were identified to species. The 1969-73 surveys were conducted in all seasons in all areas. Our survey of PRC was conducted March-May and September-November 1986, and of Pedro Bank in April 1986. Ancillary data on date, gear type, depth of trap, and habitat type (as ascertained by SCUBA) were recorded for each trap set, as in the 1969-73 survey.

The data per trap were first normalized to catch per day of soak. A least-squares regression was fitted to data from 1969-73 for the total number of fish caught per day of soak from PRC, the largest reasonably homogeneous portion of the data set:

$$
Y=0.88\left(\log _{10} \mathrm{D}\right)+0.53
$$

where $Y=$ proportion of the overall mean; and $D=$ number of days soak. An asymptote was reached after $5 \mathrm{~d}$, so for $D \geq 5, Y=1.15$. The numbers of fish caught were normalized to a $1 \mathrm{~d}$ soak by multiplying by $0.53 / \mathrm{Y}$.
For most analyses, data for the individual fish species were aggregated by family, and in several instances by size-class or to order as well, because of the extreme variability of the catches and infrequent occurrence of most species. Species comprising less than $0.1 \%$ and groups comprising less than $1 \%$ of the catch in the 1969-73 survey were eliminated. In general the dominant species comprising well-represented groups within each area did not change between surveys, although there were some notable exceptions (e.g. the queen triggerfish Balistes vetula, the dominant species trapped on SE Pedro Bank in 1969-73, was replaced by the sargassum triggerfish Xanthichthys ringens in 1986).

Several statistical procedures were used to test for differences between the 2 surveys, given possible differences in the distribution of sampling by season, sample sites (Fig. 1), habitat, or gear type between the surveys. Depth was eliminated as a factor because its effect was similar to that of habitat (i.e. reef terrace, reef slope, and reef base) but was less sensitive, since habitat depths varied between sampling sites. The data were highly non-normal due to the high incidence of zero catches for any particular species or group, and attempts to normalize the data using the logarithmic, square and cube root, and hyperbolic sine transformations. (Legendre \& Legendre 1983) were unsuccessful. Parametric analysis of variance (ANOVA) therefore was not used, and there are no non-parametric multiway ANOVA procedures appropriate for our experimental design. We therefore first examined the larger 1969-73 data sets for PRC and Pedro Bank for differences in relation to each of the above factors for each species group using Kruskal-Wallis (KW) non-parametric ANOVA. When a significant difference was found, the 1969-73 and 1986 survey data sets were subdivided by that factor. Means were calculated for the pooled data and data subsets, as well as for the 1986 data weighted by the distribution of sampling in 1969-73 among significantly different subfactors.

Differences in abundance at PRC between surveys were examined separately for each significantly different subset of data, as well as for the pooled data, using the Mann-Whitney (MW) $U$-test, a 2-sample nonparametric ANOVA (Sokal \& Rohlf 1981). Use of only data subsets may lack power to discriminate differences between the surveys (Type II error), since each subset contains only a fraction of the degrees of freedom of the original data set. We therefore looked for interaction effects - i.e. instances where the difference between the 2 surveys varied markedly depending upon the data subset being examined - and for marked differences in the distribution of sampling among subfactors between the 2 surveys. Where there was no evidence of such effects, we relied primarily upon results from the pooled data. However where such 
effects were found, significance testing was based upon random subsampling from the data sets to obtain an equal sampling distribution over the subfactor in question.

Differences between the 1969-73 and 1986 surveys of Pedro Bank could not be tested for significance using the MW test, because the earlier survey data were available only as group means (mean $=10$ trap sets per group). Due to the high incidence of zeros, data from individual trap sets and group means could not be ranked comparably for use in non-parametric statistics, and insufficient data were available from 1986 to group those data. However, the 1969-73 survey data were somewhat normalized, since they are based upon sample means, viz, the central limit theorem (Sokal \& Rohlf 1981 p. 131). The 1986 data approximated a Poisson distribution, in which the variance is equal to the mean, for which the square-root transformation is recommended (Sokal \& Rohlf 1981 p. 421-423). The pooled Pedro Bank data were therefore square-root transformed, and differences between the surveys were tested for significance using the $t$-test with the conservative assumption that variances in the 2 surveys were not equal (Sokal \& Rohlf 1981 p. 409, Norusis 1986 p. B-121).

\section{RESULTS}

Overall catch rates fell markedly in heavily fished survey areas (Tables 2 and 3, Figs. 2 and 3). Overall mean catches per trap declined most substantially over SE Pedro Bank - $76 \%$ in numbers and $82 \%$ in weight which experienced the greatest increase in fishing over the past $15 \mathrm{yr}$. Catch rates also declined significantly $38 \%$ in catch numbers and $33 \%$ in weight - in the Port Royal Cays area. On the other hand, total catch rates over lightly-to-moderately fished SW Pedro Bank were virtually unchanged between the 2 surveys. Catch rates there in numbers are now 5.5 to 6.8 times greater than in the other survey areas, and in terms of weight, catch rates are now almost 10 -fold higher. Overall catch rates in the heavily fished areas - SE Pedro Bank and PRC are now approximately equal, although in 1969-73 catches over SE Pedro Bank, then only moderately fished, were 3 -fold higher.

There are a number of similarities among areas, particularly heavily fished sites, in the changes that occurred to individual fish groups. The largest fish regularly caught in the traps, both piscivores (large serranids and lutjanids) and herbivores (large scarids) virtually disappeared from catches in all study sites. Ancillary data from visual SCUBA surveys confirmed these results: no large grouper species were observed on 27 dives along $50 \mathrm{~m}$ transects at the Port Royal sites and only 1 was observed in 24 dives at Pedro Bank
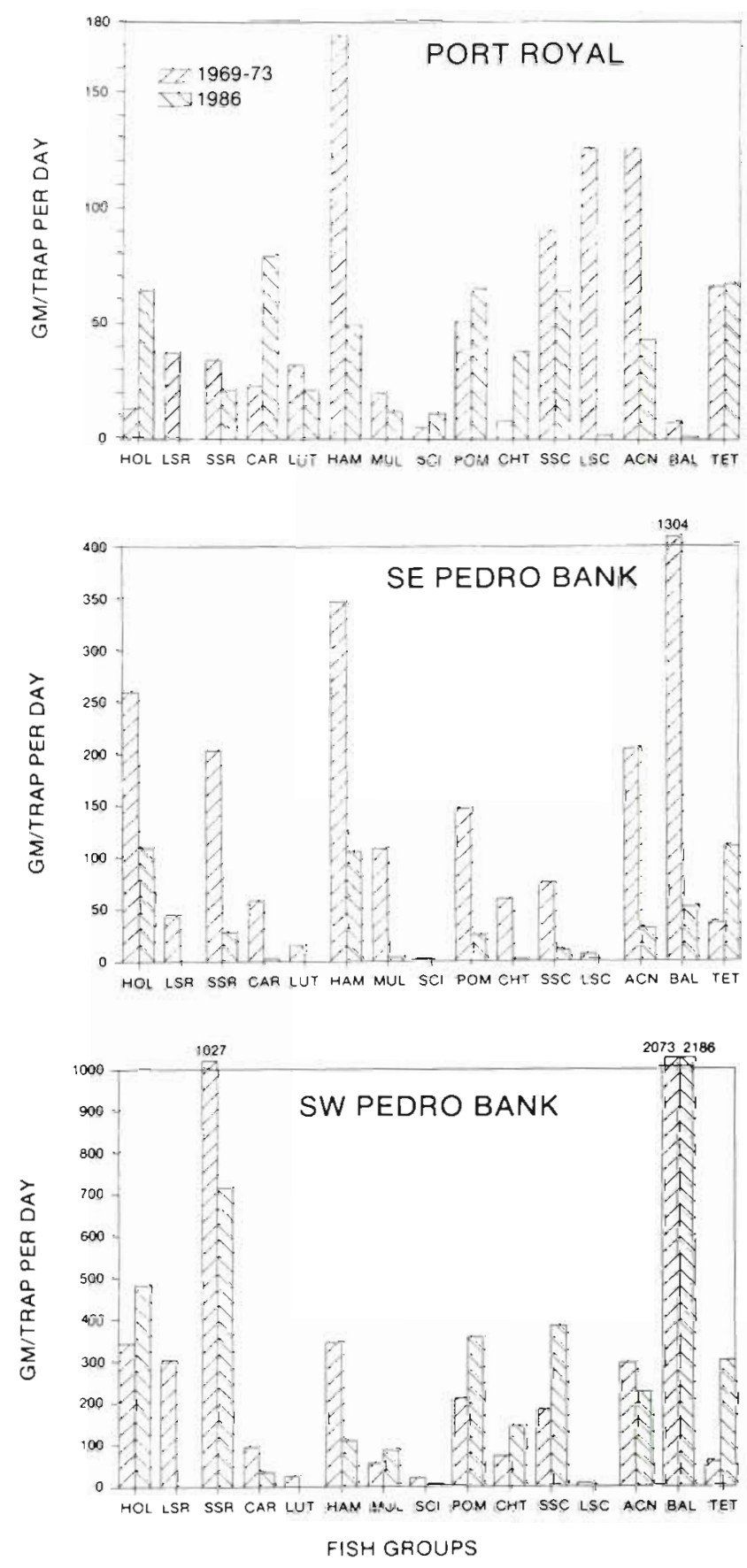

Fig. 2. $\log _{10}$-transformed mean trap catch rates $\left(g \operatorname{trap}^{-1} \mathrm{~d}^{-1}\right.$ ) for major fish groups in Port Royal Cays area, SE and SW Pedro Bank, 1969-73 and 1986. HOL: Holocentridae, LSR: large Serranidae; SSR: small Serranidae; CAR: Carangidae; LUT: Lutjanidae; HAM: Haemulidae; MUL: Mullidae; SCI: Sciaenidae; POM: Pomacanthidae; CHT: Chaetodontidae; SSC: small Scaridae; LSC: large Scaridae; ACN: Acanthuridae: BAL: Balistidae; TET: other tetraodontiformes; TOT: total

sites; and snapper and large parrot fish, except for Sparisoma viride, were uncommon in both areas $(0$ to 0.3 per dive) (Koslow unpubl.). Other commercially 

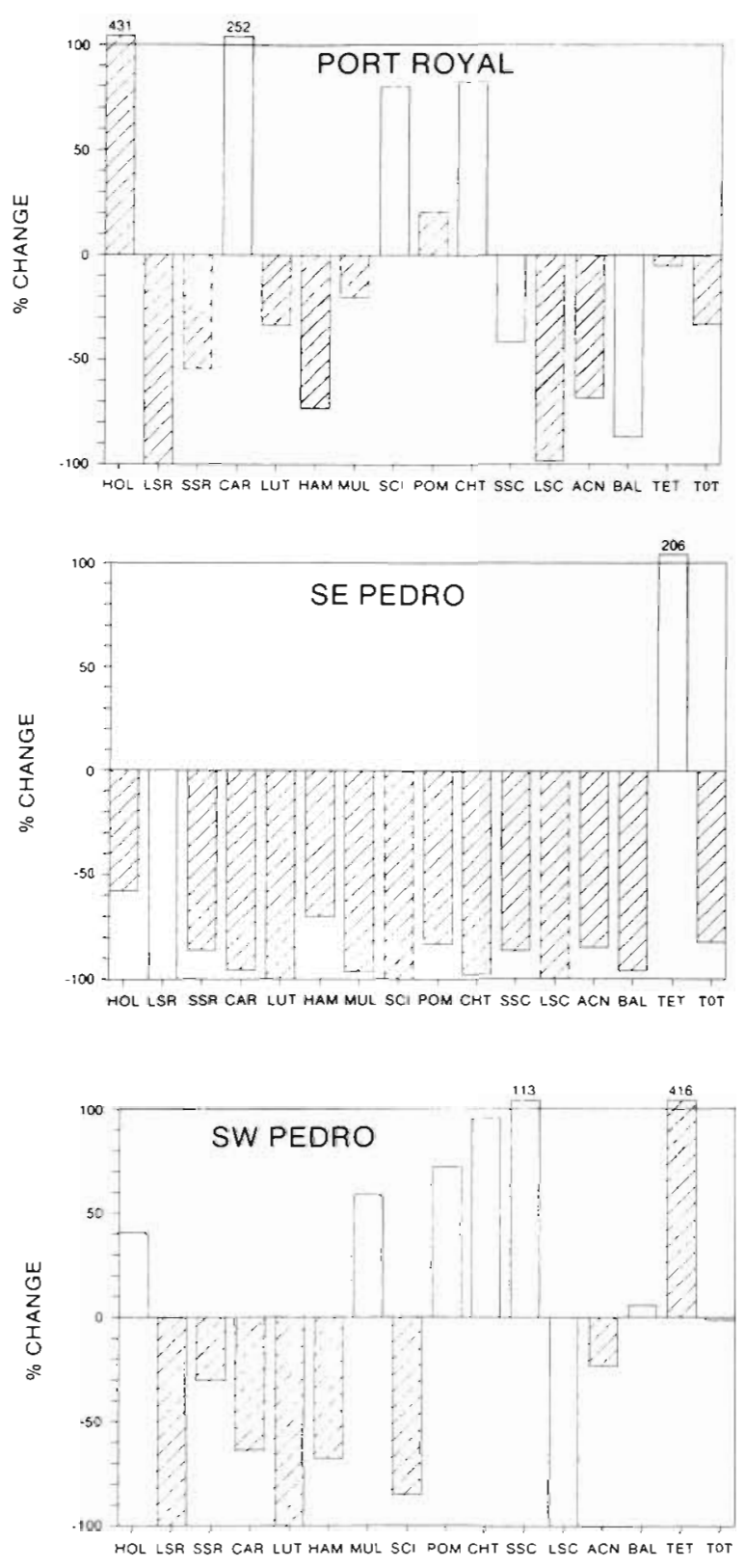

FISH GROUPS

Fig. 3. Percentage changes in catch rates $\left(g \operatorname{trap}^{-1} \mathrm{~d}^{-1}\right)$ of major fish groups between 1969-73 and 1986 surveys of Port Royal Cays area and SE and SW Pedro Bank (data from Table 3). Hatching indicates the changes are significant at $p<0.05$; a broken outline around the hatched bar indicates the difference is significant at $p<0.10$. Abbreviations for fish groups as in Fig. 2

prefered groups, such as haemulids and small serranids, generally declined as well. Deep-bodied fishes, such as pomacanthids, acanthurids and balistids also generally declined in heavily fished areas (Figs. 2 and 3). The catch of carangids increased in Port Royal the only commercially valuable group to increase in any area - but the change was not significant. Caran- gids are open-water fish and are not closely associated with the reef habitat. Moreover, length-frequency data indicate that the dominant carangid obtained at PRC, Caranx ruber, is found over these nearshore reefs while immature and that it migrates offshore upon maturity (Thompson \& Munro 1983). The only groups to increase consistently in the trap catches were so-called 'trash fish': holocentrids around PRC and non-balistid tetraodontiformes over Pedro Bank.

Significant changes in the overall composition of reef fish communities were seen at heavily exploited sites (Fig. 4). The overall composition of the trap catches over SE and SW Pedro Bank did not differ significantly in 1969-73, nor was there significant change in the catches over SW Pedro Bank between 1969-73 and 1986 (Kolmogorov-Smirnov (KS) test, $p>0.10$ ). However, the composition of the trap catches at the heavily exploited PRC and SE Pedro Bank both changed significantly between surveys (Fig. 4) (KS test, $p<0.05$ ). This reflects a shift from haemulids and acanthurids to holocentrids as the dominant trapped groups and the virtual disappearance of large scarids around PRC; and from balistids to holocentrids and non-balistid tetraodontiformes as the dominant groups in trap catches over SE Pedro Bank (Fig. 4).

\section{DISCUSSION}

Heavy trap-fishing seems to have led to a series of similar changes to the reef fish communities in 2 reef environments - exposed offshore SE Pedro Bank and protected nearshore PRC. Overall catch rates declined markedly between 1969-73 and 1986 at these 2 sites but not at lightly-fished SW Pedro Bank. Both larger (i.e. larger serranid, lutjanid and scarid) and deeper bodied (i.e. balistid, pomacanthid, and acanthurid) fishes declined disproportionately. Such fish are more susceptible to overexploitation by traps, since they may be obtained by the gear for proportionately more of their life span prior to maturity and completion of their most rapid growth. The serranids may be particularly vulnerable, because as protogynous hermaphrodites, overexploitation may seriously reduce the male population. Munro (1983) noted that in 1969-73, the male/ female ratio of 2 serranid species, which was 1:0.72 and 1:0.85 on the lightly-exploited offshore banks, was 1:5.6 and 1:6.0 at the Port Royal Cays. The fishery also competes with the larger predators by removing their prey and stimulates the yield of smaller species by removing a source of natural mortality.

SW Pedro Bank, which is subject to only light-tomoderate fishing, seemed to share many of the changes observed in more heavily exploited areas, i.e. a decline in reef-associated primary commercial species (group- 
Table 2. Mean numbers of fish per trap standardized to a 1d soak for major fish groups from 1969-73 and 1986 surveys of (a) Port Royal Cays and (b) Pedro Bank. Numbers of cases (n) and significance of difference are shown: ns, $p>0.10 ; ? p<0.10 ;{ }^{\prime} p<0.05$; $\cdots p<0.01 ; \cdots p<0.001$. If preliminary analysis of $1969-73$ data indicated catch varied significantly by subcategories of season, gear, station, or habitat, results are presented separately for each such subcategory. Means for 1986 in brackets [] are weighted by the proportion of sampling in each subcategory during 1969-73 in which catch varied significantly. Bold print indicates that differences in sampling distribution between surveys may have affected statistical analyses: statistics were redone based upon random subsampling from the surveys to obtain an equal sampling distribution. Results are given beside the weighted means. Subcategories of season are not shown if sampling in 1986 was not conducted then. Seasons: 1, Months 12,1,2; 2, Months 3-5; 3, Months 6-8; 4, Months 9-11. Traps: 1, unbaited Z: 2, baited stackable. Station: N, Nassau Reef; Y, Yahoo Reef; E, E Pedro (SE Spur \& Pedro Cays) ; W, W Pedro (20 m Bank \& SW Tip). Habitat: 1, Reef crest; 2, Reef slope; 3, Reef base

\begin{tabular}{|c|c|c|c|c|c|c|c|c|c|c|}
\hline \multirow{2}{*}{$\begin{array}{l}\text { (a) Port Royal } \\
\text { Fish group }\end{array}$} & \multicolumn{2}{|c|}{ All cases } & \multicolumn{2}{|c|}{ Season } & \multicolumn{2}{|c|}{ Station } & \multicolumn{2}{|c|}{ Habitat } & \multicolumn{2}{|c|}{ Trap type } \\
\hline & $1969-73$ & 1986 & $1969-73$ & 1986 & $1969-73$ & 1986 & $1969-73$ & 1986 & $1969-73$ & 1986 \\
\hline $\begin{array}{l}\text { Holocentridae } \\
\qquad(n)\end{array}$ & $\begin{array}{r}0.15 \\
(663)\end{array}$ & $\begin{array}{c}0.60 \cdots \\
{[0.55]} \\
(169)\end{array}$ & $\begin{array}{r}2-4: 0.15 \\
(571)\end{array}$ & $\begin{array}{c}0.60 \cdots \\
(169)\end{array}$ & $\begin{array}{r}N: 0.22 \\
(333) \\
Y: 0.08 \\
(330)\end{array}$ & $\begin{array}{c}0.81^{\circ} \\
(100) \\
\left(0.29^{\circ}\right. \\
(69)\end{array}$ & & & & \\
\hline $\begin{array}{l}\text { Serranidae } \\
\text { (large) }\end{array}$ & $\begin{array}{r}0.02 \\
(663)\end{array}$ & $\begin{array}{c}0.00^{*} \\
{[0.00]} \\
(169)\end{array}$ & $\begin{array}{r}2: 0.03 \\
(159) \\
1,3,4: 0.01 \\
(504)\end{array}$ & $\begin{array}{l}0.00 \mathrm{~ns} \\
(25) \\
0.00 \mathrm{~ns} \\
(144)\end{array}$ & & & & & & \\
\hline $\begin{array}{l}\text { Serranidae } \\
\text { (small) } \\
\end{array}$ & $\begin{array}{r}0.15 \\
(663)\end{array}$ & $\begin{array}{l}0.07 \\
{[0.10) \mathrm{ns}} \\
(169)\end{array}$ & $\begin{array}{r}1,4: 0.09 \\
(338) \\
2,3: 0.21 \\
(325)\end{array}$ & $\begin{array}{l}0.05^{\cdots} \\
(144) \\
0.18 \mathrm{~ns} \\
(25)\end{array}$ & $\begin{array}{r}N: 0.12 \\
(333) \\
Y: 0.19 \\
(330)\end{array}$ & $\begin{array}{c}0.08 ? \\
(100) \\
0.06 \cdots \\
(69)\end{array}$ & $\begin{array}{r}1,2: 0.15 \\
(636) \\
3: 0.05 \\
(27)\end{array}$ & $\begin{array}{l}0.13 \mathrm{~ns} \\
(80) \\
0.02 \mathrm{~ns} \\
(89)\end{array}$ & & \\
\hline $\begin{array}{l}\text { Carangidae } \\
\qquad(n)\end{array}$ & $\begin{array}{r}0.13 \\
(69-73>86) \\
(663)\end{array}$ & $\begin{array}{c}0.25 \\
{[0.18] ?} \\
(169)\end{array}$ & $\begin{array}{r}1,2,4: 0.15 \\
(497)\end{array}$ & $\begin{array}{l}0.25 \mathrm{~ns} \\
(169)\end{array}$ & & & $\begin{array}{r}1: 0.08 \\
(266) \\
2,3: 0.15 \\
(397)\end{array}$ & $\begin{array}{l}0.03 \mathrm{~ns} \\
(17) \\
0.28 \mathrm{~ns} \\
(152)\end{array}$ & & \\
\hline $\begin{array}{l}\text { Lutjanidae } \\
\qquad(n)\end{array}$ & $\begin{array}{r}0.15 \\
(663)\end{array}$ & $\begin{array}{l}0.07^{\circ} \\
(169)\end{array}$ & & & & & & & $\begin{array}{r}1: 0.17 \\
(361)\end{array}$ & $\begin{array}{l}0.07^{\cdots} \\
(169)\end{array}$ \\
\hline $\begin{array}{l}\text { Haemulidae } \\
\qquad(n)\end{array}$ & $\begin{array}{l}1.26 \\
(663)\end{array}$ & $\begin{array}{l}0.23 \cdots \\
{[0.25]} \\
(169)\end{array}$ & $\begin{array}{r}1,2,4: 1.09 \\
(497)\end{array}$ & $\begin{array}{c}0.23^{\cdots} \\
(169)\end{array}$ & $\begin{array}{r}N: 1.33 \\
(333) \\
Y: 1.20 \\
(330)\end{array}$ & $\begin{array}{c}0.21 \cdots \\
(100) \\
0.30^{\cdots} \cdots \\
(69)\end{array}$ & & & & \\
\hline Mullidae & $\begin{array}{l}0.14 \\
(663)\end{array}$ & $\begin{array}{c}0.10 \\
{[0.08]^{*}} \\
(169)\end{array}$ & $\begin{array}{r}1,2,4: 0.17 \\
(497)\end{array}$ & $\begin{array}{l}0.10 \mathrm{~ns} \\
(169)\end{array}$ & $\begin{array}{r}N: 0.22 \\
(333) \\
Y: 0.06 \\
(330)\end{array}$ & $\begin{array}{l}0.15 \mathrm{~ns} \\
(100) \\
0.02 \mathrm{~ns} \\
(69)\end{array}$ & $\begin{array}{r}1: 0.05 \\
(266) \\
2,3: 0.20 \\
{[397)}\end{array}$ & $\begin{array}{l}0.00 \mathrm{~ns} \\
(17) \\
0.11 ? \\
(152)\end{array}$ & $\begin{array}{r}1: 0.08 \\
(361)\end{array}$ & $\begin{array}{l}0.10 \mathrm{~ns} \\
(169)\end{array}$ \\
\hline Sciaenidae & $\begin{array}{r}0.04 \\
(663)\end{array}$ & $\begin{array}{l}0.06 \\
{[0.08] \mathrm{ns}} \\
(169)\end{array}$ & & & & & $\begin{array}{r}1,2: 0.04 \\
(636) \\
3: 0.03 \\
(27)\end{array}$ & $\begin{array}{l}0.08 \mathrm{~ns} \\
(80) \\
0.04 \mathrm{~ns} \\
(89)\end{array}$ & & \\
\hline $\begin{array}{l}\text { Pomacanthidae } \\
\qquad(n)\end{array}$ & $\begin{array}{l}0.16 \\
(663)\end{array}$ & $\begin{array}{c}0.14 \\
{[0.15]^{\cdots}} \\
(169)\end{array}$ & & & $\begin{array}{r}\mathrm{N}: 0.07 \\
(333) \\
\mathrm{Y}: 0.24 \\
(330)\end{array}$ & $\begin{array}{c}0.08 \mathrm{~ns} \\
(100) \\
0.23^{\circ} \\
(69)\end{array}$ & & & & \\
\hline $\begin{array}{l}\text { Chaetodontidae } \\
\qquad(n)\end{array}$ & $\begin{array}{l}0.31 \\
(663)\end{array}$ & $\begin{array}{l}0.24 \\
{[0.33] \mathrm{ns}} \\
(169)\end{array}$ & & & & & $\begin{array}{r}1: 0.40 \\
(266) \\
2,3: 0.24 \\
(397)\end{array}$ & $\begin{array}{l}0.51 ? \\
(17) \\
0.21 \mathrm{~ns} \\
(152)\end{array}$ & & \\
\hline $\begin{array}{l}\text { Scaridae } \\
\text { (small) }\end{array}$ & $\begin{array}{r}0.70 \\
(663)\end{array}$ & $\begin{array}{l}0.40 \\
{[0.49] \mathrm{ns}} \\
(169)\end{array}$ & $\begin{array}{r}2: 1.02 \\
(159) \\
1,3,4: 0.60 \\
(504)\end{array}$ & $\begin{array}{l}0.74 \mathrm{~ns} \\
(25) \\
0.34 \cdots \\
(144)\end{array}$ & $\begin{array}{r}N: 0.68 \\
(333) \\
Y: 0.73 \\
(330)\end{array}$ & $\begin{array}{c}0.40^{\circ} \\
(100) \\
0.40^{\circ} \\
(69)\end{array}$ & $\begin{array}{r}1,2: 0.73 \\
(636) \\
3: 0.17 \\
(27)\end{array}$ & $\begin{array}{l}0.65 \mathrm{~ns} \\
(80) \\
0.17 \mathrm{~ns} \\
(89)\end{array}$ & & \\
\hline $\begin{array}{l}\text { Scaridae } \\
\text { (large) }\end{array}$ & $\begin{array}{r}0.65 \\
(663)\end{array}$ & $\begin{array}{c}0.01 \cdots \\
{[0.01]} \\
(169)\end{array}$ & & & $\begin{array}{r}N: 1.00 \\
(333) \\
Y: 0.30 \\
(330)\end{array}$ & $\begin{array}{l}0.00 \cdots \\
(100) \\
0.02 \cdots \\
(69)\end{array}$ & $\begin{array}{r}1: 0.75 \\
(266) \\
2: 0.63 \\
(370) \\
3: 0.02 \\
(27)\end{array}$ & $\begin{array}{l}0.00 \cdots \\
(17) \\
0.02 \cdots \\
(63) \\
0.00 ? \\
(89)\end{array}$ & & \\
\hline
\end{tabular}


Table 2 (continued)

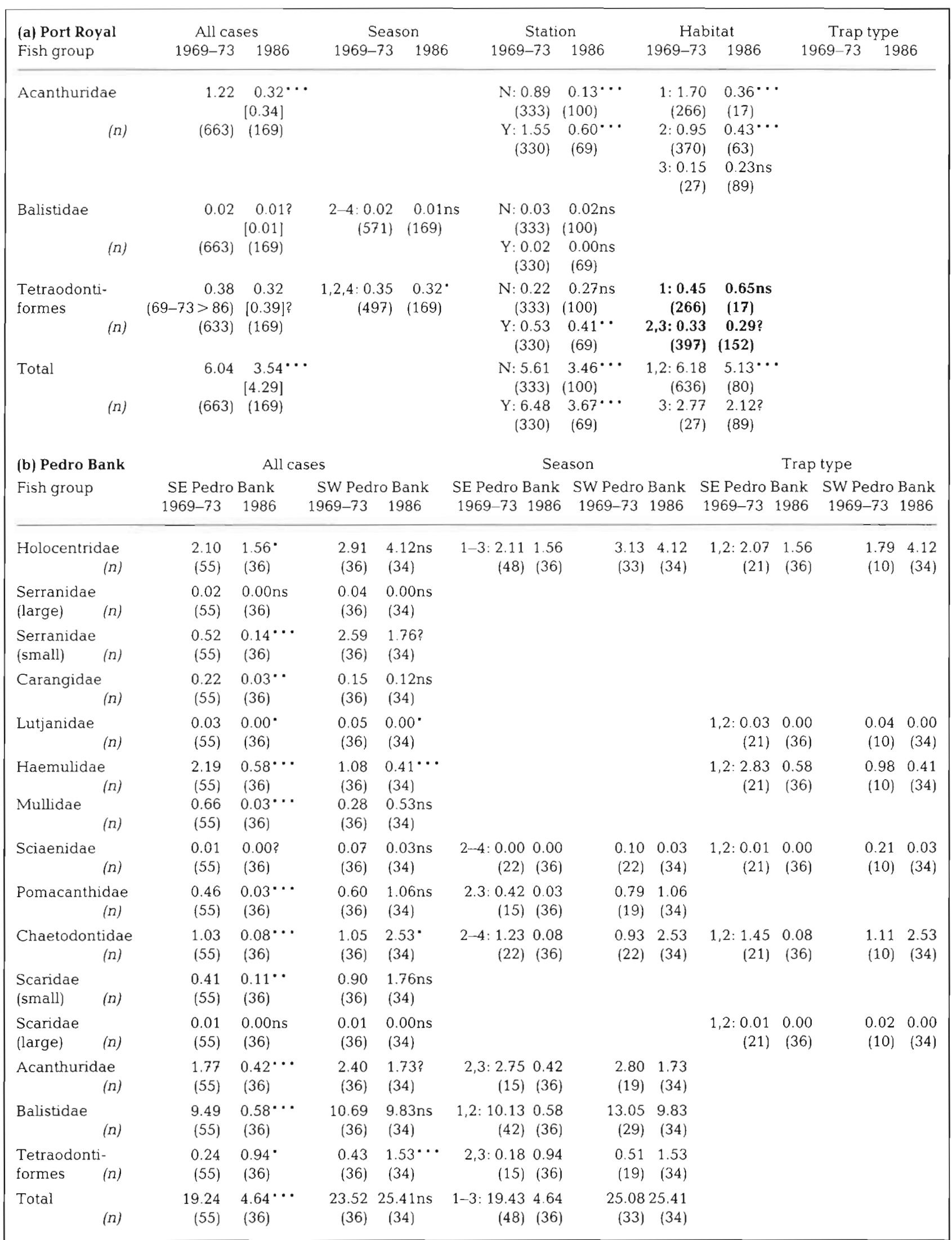


Table 3. Mean catch rate $(\mathrm{g})$ of fish per trap standardized to a $1 \mathrm{~d}$ soak for major fish groups from 1969-73 and 1986 surveys of (a) Port Royal Cays and (b) Pedro Bank. Numbers of cases (n) and significance of difference are shown: ns, $p>0.10 ; ? p<0.10 ; \cdot p<0.05$; $\cdots p<0.01 ; \cdots p<0.001$. If preliminary analysis of 1969-73 data indicated catch varied significantly by subcategories of season, gear, station, or habitat, results are presented separately for each such subcategory. The means for 1986 in brackets [ ] are weighted by the proportion of sampling in each subcategory during 1969-73 in which catch varied significantly. Bold print indicates that differences in sampling distribution between surveys may have affected statistical analyses: statistics were redone based upon random subsampling from the surveys to obtain an equal sampling distribution. Results are given beside the weighted means. Subcategories of season are not shown if sampling in 1986 was not conducted then. Seasons: 1 , Months $12,1,2 ; 2$, Months $3-5 ; 3$, Months 6-8; 4, Months 9-11. Traps: 1, unbaited Z; 2, baited stackable. Station: N, Nassau Reef; Y, Yahoo Reef; E, E Pedro (SE Spur \& Pedro Cays); W, W Pedro (20 m Bank \& SW Tip). Habitat: 1, reef crest; 2, reef slope; 3, reef base

\begin{tabular}{|c|c|c|c|c|c|c|c|c|c|c|}
\hline \multirow{2}{*}{$\begin{array}{l}\text { (a) Port Royal } \\
\text { Fish groups }\end{array}$} & \multicolumn{2}{|c|}{ All cases } & \multicolumn{2}{|c|}{ Season } & \multicolumn{2}{|c|}{ Station } & \multicolumn{2}{|c|}{ Habitat } & \multicolumn{2}{|c|}{ Trap type } \\
\hline & $1969-73$ & 1986 & $1969-73$ & 1986 & $1969-73$ & 1986 & $1969-73$ & 1986 & $1969-73$ & 1986 \\
\hline $\begin{array}{l}\text { Holocentridae } \\
\qquad(n)\end{array}$ & $\begin{array}{r}13 \\
(663)\end{array}$ & $\begin{array}{l}69 \cdots \\
{[64]} \\
(166)\end{array}$ & $\begin{array}{r}2-4: 13 \\
(570)\end{array}$ & $\begin{array}{c}69^{\cdots} \\
(166)\end{array}$ & $\begin{array}{r}N: 19 \\
(333) \\
Y: 8 \\
(330)\end{array}$ & $\begin{array}{c}94^{\cdots} \\
(97) \\
34^{\circ} \\
(69)\end{array}$ & & & & \\
\hline $\begin{array}{l}\text { Serranidae } \\
\text { (large) }\end{array}$ & $\begin{array}{r}37 \\
(663)\end{array}$ & $\begin{array}{r}0^{*} \\
{[0]} \\
(166)\end{array}$ & $\begin{array}{r}2: 119 \\
(158) \\
1,3,4: 12 \\
(504)\end{array}$ & $\begin{array}{c}\text { Ons } \\
(21) \\
\text { Ons } \\
(145)\end{array}$ & & & & & & \\
\hline $\begin{array}{l}\text { Serranidae } \\
\text { (Small) } \\
\end{array}$ & $\begin{array}{r}34 \\
(663)\end{array}$ & $\begin{array}{c}15 \\
{[21) ?} \\
(166)\end{array}$ & $\begin{array}{r}1,4: 22 \\
(338) \\
2,3: 47 \\
(325)\end{array}$ & $\begin{array}{c}15^{\cdots} \\
(144) \\
22 \mathrm{~ns} \\
(25)\end{array}$ & $\begin{array}{l}N: 28 \\
(333) \\
Y: 40 \\
(330)\end{array}$ & $\begin{array}{c}15^{\circ} \\
(97) \\
16^{\cdots} \\
(69)\end{array}$ & $\begin{array}{r}1,2: 35 \\
(636) \\
3: 15 \\
(27)\end{array}$ & $\begin{array}{l}29 \mathrm{~ns} \\
(78) \\
4 \mathrm{~ns} \\
(88)\end{array}$ & & \\
\hline $\begin{array}{l}\text { Carangidae } \\
\qquad(n)\end{array}$ & $\begin{array}{r}23 \\
(662)\end{array}$ & $\begin{array}{c}81 \\
{[79] \mathrm{ns}} \\
(166)\end{array}$ & $\begin{array}{r}1,2,4: 27 \\
(496)\end{array}$ & $\begin{array}{c}81 \mathrm{~ns} \\
(166)\end{array}$ & & & $\begin{array}{r}1: 9 \\
(266) \\
2,3: 33 \\
(396)\end{array}$ & $\begin{array}{c}75 \mathrm{~ns} \\
(16) \\
81 \mathrm{~ns} \\
(150)\end{array}$ & & \\
\hline $\begin{array}{l}\text { Lutjanidae } \\
\qquad(n)\end{array}$ & $\begin{array}{r}32 \\
(663)\end{array}$ & $\underset{(166)}{21^{\cdots}}$ & & & & & & & $\begin{array}{l}1: 34 \\
(361)\end{array}$ & $\begin{array}{c}21^{\cdots} \\
(166)\end{array}$ \\
\hline $\begin{array}{l}\text { Haemulidae } \\
\qquad(n)\end{array}$ & $\begin{array}{r}174 \\
(661)\end{array}$ & $\begin{array}{r}46^{\cdots} \\
{[49]} \\
(166)\end{array}$ & $\begin{array}{r}1,2,4: 153 \\
(496)\end{array}$ & $\begin{array}{r}46^{\cdots} \\
(166)\end{array}$ & $\begin{array}{r}N: 166 \\
(332) \\
Y \cdot 183 \\
(329)\end{array}$ & $\begin{array}{c}35^{\cdots} \\
(97) \\
62 \cdots \\
(69)\end{array}$ & & & & \\
\hline Mullidae & $\begin{array}{r}20 \\
(662)\end{array}$ & $\begin{array}{r}16 \\
{[12]^{\circ}} \\
(166)\end{array}$ & $\begin{array}{r}1,2,4: 24 \\
(496)\end{array}$ & $\begin{array}{c}16 \text { ns } \\
(166)\end{array}$ & $\begin{array}{l}N: 31 \\
(332) \\
Y: 10 \\
(330)\end{array}$ & $\begin{array}{l}25 \mathrm{~ns} \\
(97) \\
3 \mathrm{~ns} \\
(69)\end{array}$ & $\begin{array}{r}1: 7 \\
(266) \\
2,3: 30 \\
(396)\end{array}$ & $\begin{array}{c}\text { Ons } \\
(16) \\
0.18 ? \\
(150)\end{array}$ & $\begin{array}{l}1: 12 \\
(360)\end{array}$ & $\begin{array}{c}16 \mathrm{~ns} \\
(166)\end{array}$ \\
\hline Sciaenidae & $\begin{array}{r}5 \\
(663)\end{array}$ & $\begin{array}{c}9 \\
{[11] \mathrm{ns}} \\
(166)\end{array}$ & & & & & $\begin{array}{r}1,2: 5 \\
(636) \\
3: 4 \\
(27)\end{array}$ & $\begin{array}{c}11 \mathrm{~ns} \\
(78) \\
8 \mathrm{~ns} \\
(88)\end{array}$ & & \\
\hline $\begin{array}{l}\text { Pomacanthidae } \\
\qquad(n)\end{array}$ & $\begin{array}{r}51 \\
(663)\end{array}$ & $\begin{array}{c}61 \\
{[65]^{*}} \\
(166)\end{array}$ & & & $\begin{array}{l}N: 14 \\
(333) \\
Y: 88 \\
(330)\end{array}$ & $\begin{array}{c}40 \mathrm{~ns} \\
(97) \\
91^{\circ} \\
(69)\end{array}$ & & & & \\
\hline $\begin{array}{l}\text { Chaetodontidae } \\
\qquad(n)\end{array}$ & $\begin{array}{r}8 \\
(663)\end{array}$ & $\begin{array}{c}15 \\
{[38] \mathrm{ns}} \\
(166)\end{array}$ & & & & & $\begin{array}{l}1: 11 \\
(267) \\
2,3: 6 \\
(396)\end{array}$ & $\begin{array}{c}85 \mathrm{~ns} \\
(16) \\
7 n s \\
(150)\end{array}$ & & \\
\hline $\begin{array}{l}\text { Scaridae } \\
\text { (small) }\end{array}$ & $\begin{array}{r}90 \\
\{662\}\end{array}$ & $\begin{array}{c}52 \\
{[64] \mathrm{ns}} \\
(166)\end{array}$ & $\begin{array}{r}2: 136 \\
(158) \\
1,3,4: 76 \\
(504)\end{array}$ & $\begin{array}{c}84 \mathrm{~ns} \\
(21) \\
47 \cdots \\
(145)\end{array}$ & $\begin{array}{l}N: 96 \\
(332) \\
Y: 84 \\
(330)\end{array}$ & $\begin{array}{c}47^{\circ} \\
(97) \\
60^{\circ} \\
(69)\end{array}$ & $\begin{array}{r}1,2: 92 \\
(635) \\
3: 34 \\
(27)\end{array}$ & $\begin{array}{c}84 \mathrm{~ns} \\
(78) \\
24 \mathrm{~ns} \\
(88)\end{array}$ & & \\
\hline $\begin{array}{l}\text { Scaridae } \\
\text { (large) }\end{array}$ & $\begin{array}{r}125 \\
(662)\end{array}$ & $\begin{array}{r}2 \cdots \\
{[2]} \\
(166)\end{array}$ & & & $\begin{array}{r}N: 185 \\
(332) \\
Y: 64 \\
(330)\end{array}$ & $\begin{array}{c}0 \cdots \\
(97) \\
4 \cdots \\
(69)\end{array}$ & $\begin{array}{r}1.145 \\
(267) \\
2: 119 \\
(368) \\
3: 2 \\
(27)\end{array}$ & $\begin{array}{c}0 \cdots \\
(16) \\
4 \cdots \\
(62) \\
0 ? \\
(88)\end{array}$ & & \\
\hline
\end{tabular}


Table 3 (continued)

\begin{tabular}{|c|c|c|c|c|c|c|c|c|c|c|c|}
\hline \multirow{2}{*}{$\begin{array}{l}\text { (a) Port Royal } \\
\text { Fish group }\end{array}$} & \multicolumn{2}{|c|}{ All cases } & \multicolumn{2}{|c|}{ Season } & \multicolumn{2}{|c|}{ Station } & \multicolumn{2}{|c|}{ Habitat } & \multicolumn{3}{|c|}{ Trap type } \\
\hline & $1969-73$ & 1986 & \multicolumn{2}{|c|}{$1969-73 \quad 1986$} & \multicolumn{2}{|c|}{$1969-73 \quad 1986$} & \multicolumn{2}{|c|}{$1969-73 \quad 1986$} & \multicolumn{3}{|c|}{$1969-73 \quad 1986$} \\
\hline $\begin{array}{l}\text { Acanthuridae } \\
\qquad(n)\end{array}$ & $\begin{array}{r}125 \\
(663)\end{array}$ & $\begin{array}{c}39 \cdots \\
{[43]} \\
(166)\end{array}$ & & & $\begin{array}{r}N: 71 \\
(333) \\
Y: 179 \\
(330)\end{array}$ & $\begin{array}{c}11^{\cdots} \cdots \\
(97) \\
79^{\cdots} \cdots \\
(69)\end{array}$ & $\begin{array}{r}116 \\
(267 \\
2: 10 \\
(369 \\
3: 1 \\
(27\end{array}$ & $\begin{array}{cc}9 & 17 \cdots \\
7) & (16) \\
1 & 60 \cdots \\
9) & (62) \\
8 & 29 \mathrm{~ns} \\
7) & (88)\end{array}$ & & & \\
\hline Balistidae & $\begin{array}{r}7 \\
(663)\end{array}$ & $\begin{array}{r}1 \mathrm{~ns} \\
{[1]} \\
(166)\end{array}$ & $\begin{array}{r}2-4: \\
(57\end{array}$ & $\begin{array}{rr}7 & 11 \\
0) & (166)\end{array}$ & $\begin{array}{r}N: 8 \\
(333) \\
Y: 7 \\
(330)\end{array}$ & $\begin{array}{l}2 \mathrm{~ns} \\
(97)^{0 n s} \\
(69)\end{array}$ & & & & & \\
\hline $\begin{array}{l}\text { Tetraodonti- } \\
\text { formes } \\
\qquad(n)\end{array}$ & $\begin{array}{r}66 \\
(69-73>86) \\
(663)\end{array}$ & $\begin{array}{c}62 \\
{[67]^{\circ}} \\
(166)\end{array}$ & $\begin{array}{r}1,2,4: 6 \\
(49\end{array}$ & $\begin{array}{rr}66 & 62 \\
7) & (166)\end{array}$ & $\begin{array}{l}N: 37 \\
(333) \\
Y: 96 \\
(330)\end{array}$ & $\begin{array}{c}43 \mathrm{~ns} \\
(97) \\
89^{\circ} \\
(69)\end{array}$ & $\begin{array}{r}1: 7 \\
\{267 \\
2,3: 5 \\
(396\end{array}$ & $\begin{array}{lc}7 & 78 \mathrm{~ns} \\
7) & (16) \\
9 & 61 \mathrm{~ns} \\
6 & (150)\end{array}$ & & & \\
\hline Total & $\begin{array}{r}876 \\
(657)\end{array}$ & $\begin{array}{l}588^{*} \\
{[642]} \\
(166)\end{array}$ & & & $\begin{array}{r}\text { N: } 822 \\
(329) \\
Y: 930 \\
(328)\end{array}$ & $\begin{array}{l}518^{\cdots} \cdots \\
(97) \\
686^{\cdots} \cdots \\
(69)\end{array}$ & $\begin{array}{r}1,2: 89 \\
1630 \\
3: 45 \\
(27\end{array}$ & $\begin{array}{ll}4 & 689 \cdots \\
0) & (78) \\
5 & 498 \mathrm{~ns} \\
7) & (88)\end{array}$ & & & \\
\hline \multirow{2}{*}{$\begin{array}{l}\text { (b) Pedro Bank } \\
\text { Fish group }\end{array}$} & \multicolumn{4}{|c|}{ All cases } & \multicolumn{3}{|c|}{ Season } & \multicolumn{4}{|c|}{ Trap type } \\
\hline & $\begin{array}{l}\text { SE Pedro } \\
1969-73\end{array}$ & $\begin{array}{r}\text { Bank } \\
1986\end{array}$ & $\begin{array}{l}\text { SW Pedr } \\
1969-73\end{array}$ & $\begin{array}{l}\text { o Bank } \\
1986\end{array}$ & $\begin{array}{c}\text { SE Pedro Bank } \\
1969-73 \quad 1986\end{array}$ & $\begin{array}{c}\text { SW Pedro } \\
1969-73\end{array}$ & $\begin{array}{l}\text { Bank } \\
1986\end{array}$ & $\begin{array}{l}\text { SE Pedro } \mathrm{B} \\
1969-73\end{array}$ & $\begin{array}{l}\text { Bank } \\
1986\end{array}$ & $\begin{array}{c}\text { SW Pedro } \\
1969-73\end{array}$ & $\begin{array}{l}\text { Bank } \\
1986\end{array}$ \\
\hline $\begin{array}{r}\text { Holocentridae } \\
\text { (n) }\end{array}$ & $\begin{array}{l}260 \\
(55)\end{array}$ & $\begin{array}{l}109 \cdots \\
(36)\end{array}$ & $\begin{array}{l}342 \\
(36)\end{array}$ & $\begin{array}{l}\text { 481ns } \\
(34)\end{array}$ & 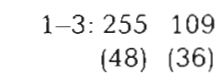 & $\begin{array}{l}368 \\
(33)\end{array}$ & $\begin{array}{l}481 \\
(34)\end{array}$ & $\begin{array}{r}1,2: 258 \\
(21)\end{array}$ & $\begin{array}{l}109 \\
(36)\end{array}$ & $\begin{array}{l}191 \\
(10)\end{array}$ & $\begin{array}{l}481 \\
(34)\end{array}$ \\
\hline $\begin{array}{l}\text { Serranidae } \\
\text { (large) (n) }\end{array}$ & $\begin{array}{r}44 \\
(55)\end{array}$ & $\begin{array}{l}\text { Ons } \\
(36)\end{array}$ & $\begin{array}{l}300 \\
(36)\end{array}$ & $\begin{array}{c}0^{\circ} \\
(34)^{\circ}\end{array}$ & & & & & & & \\
\hline $\begin{array}{l}\text { Serranidae } \\
\text { (small) (n) }\end{array}$ & $\begin{array}{l}202 \\
(55)\end{array}$ & $\begin{array}{l}28 \cdots \\
(36)\end{array}$ & $\begin{array}{r}1027 \\
(36)\end{array}$ & $\begin{array}{l}715^{\circ} \\
(34)\end{array}$ & & & & & & & \\
\hline $\begin{array}{r}\text { Carangidae } \\
\text { (n) }\end{array}$ & $\begin{array}{r}58 \\
(55)\end{array}$ & $\begin{array}{l}3 \cdots \\
(36)\end{array}$ & $\begin{array}{r}95 \\
(36)\end{array}$ & $\begin{array}{r}34^{\circ} \\
(34)\end{array}$ & & & & & & & \\
\hline $\begin{array}{l}\text { Lutjanidae } \\
\qquad(n)\end{array}$ & $\begin{array}{r}15 \\
(55)\end{array}$ & $\begin{array}{l}0^{*} \\
(36)\end{array}$ & $\begin{array}{r}24 \\
(36)\end{array}$ & $\begin{array}{c}0^{\cdots} \\
(34)\end{array}$ & & & & $\begin{array}{r}1,2: 20 \\
(21)\end{array}$ & $\begin{array}{r}0 \\
(36)\end{array}$ & $\begin{array}{r}22 \\
(10)\end{array}$ & $\begin{array}{r}0 \\
(34)\end{array}$ \\
\hline $\begin{array}{l}\text { Haemulidae } \\
\qquad(n)\end{array}$ & $\begin{array}{r}347 \\
(55)\end{array}$ & $\begin{array}{l}105^{\cdots} \\
(36)\end{array}$ & $\begin{array}{l}342 \\
(36)\end{array}$ & $\begin{array}{l}110^{\cdots} \cdots \\
(34)\end{array}$ & & & & $\begin{array}{r}1.2: 454 \\
(21)\end{array}$ & $\begin{array}{l}105 \\
(36)\end{array}$ & $\begin{array}{r}214 \\
(10)\end{array}$ & $\begin{array}{l}110 \\
(34)\end{array}$ \\
\hline $\begin{array}{l}\text { Mullidae } \\
\text { (n) }\end{array}$ & $\begin{array}{l}108 \\
(55)\end{array}$ & $\begin{array}{l}3 \cdots \\
(36)\end{array}$ & $\begin{array}{r}55 \\
(36)\end{array}$ & $\begin{array}{l}87 \mathrm{~ns} \\
(34)\end{array}$ & & & & & & & \\
\hline $\begin{array}{l}\text { Sciaenidae } \\
\text { (n) }\end{array}$ & $\begin{array}{r}1 \\
(55)\end{array}$ & $\begin{array}{c}0 ? \\
(36)\end{array}$ & $\begin{array}{r}20 \\
(36)\end{array}$ & $3^{3 \cdots}$ & $\begin{array}{rr}2-4: 1 & 0 \\
(22) & (36)\end{array}$ & $\begin{array}{r}27 \\
(22)\end{array}$ & $\begin{array}{r}3 \\
(34)\end{array}$ & $\begin{array}{r}1,2: 3 \\
(21)\end{array}$ & $\begin{array}{r}0 \\
(36)\end{array}$ & $\begin{array}{r}60 \\
(10)\end{array}$ & $\begin{array}{r}3 \\
(34)\end{array}$ \\
\hline $\begin{array}{r}\text { Pomacanthidae } \\
\text { (n) }\end{array}$ & $\begin{array}{l}146 \\
(55)\end{array}$ & $\begin{array}{l}25 \cdots \\
(36)\end{array}$ & $\begin{array}{l}207 \\
(36)\end{array}$ & $\begin{array}{l}356 \mathrm{~ns} \\
(34)\end{array}$ & $\begin{array}{rrr}2,3: & 126 & 25 \\
(15) & (36)\end{array}$ & $\begin{array}{l}274 \\
(19)\end{array}$ & $\begin{array}{l}356 \\
(34)\end{array}$ & & & & \\
\hline $\begin{array}{c}\text { Chaetodontidae } \\
\text { (n) }\end{array}$ & $\begin{array}{r}60 \\
(55)\end{array}$ & $\frac{1 \cdots}{(36)}$ & $\begin{array}{r}73 \\
(36)\end{array}$ & $\begin{array}{l}143 \mathrm{~ns} \\
(34)\end{array}$ & $\begin{array}{rr}2-4: 74 & 1 \\
(22) & (36)\end{array}$ & $\begin{array}{r}68 \\
(22)\end{array}$ & 143 & $\begin{array}{r}1,2: 84 \\
(21)\end{array}$ & $\begin{array}{r}1 \\
(36)\end{array}$ & $\begin{array}{r}90 \\
(10)\end{array}$ & $\begin{array}{r}143 \\
(34)\end{array}$ \\
\hline $\begin{array}{l}\text { Scaridae } \\
\text { (small) (n) }\end{array}$ & $\begin{array}{r}74 \\
(55)\end{array}$ & $\begin{array}{l}10^{\cdots} \\
(36)\end{array}$ & $\begin{array}{l}179 \\
(36)\end{array}$ & $\begin{array}{l}382 \mathrm{~ns} \\
(34)\end{array}$ & & & & & & & \\
\hline $\begin{array}{l}\text { Scaridae } \\
\text { (large) }\end{array}$ & $\begin{array}{r}6 \\
(55)\end{array}$ & $\begin{array}{c}0 ? \\
(36)\end{array}$ & $\begin{array}{r}7 \\
(36)\end{array}$ & $\begin{array}{l}\text { Ons } \\
(34)\end{array}$ & & & & $\begin{array}{r}1,2: 9 \\
(21)\end{array}$ & $\begin{array}{r}0 \\
(36)\end{array}$ & $\begin{array}{r}21 \\
(10)\end{array}$ & $\begin{array}{r}0 \\
(34)\end{array}$ \\
\hline $\begin{array}{r}\text { Acanthuridae } \\
\text { (n) }\end{array}$ & $\begin{array}{l}204 \\
(55)\end{array}$ & $\begin{array}{l}31 \cdots \\
(36)\end{array}$ & $\begin{array}{l}293 \\
(36)\end{array}$ & $\begin{array}{l}224^{\circ} \\
(34)\end{array}$ & $\begin{array}{rr}2,3: 293 & 31 \\
(15) & (36)\end{array}$ & $\begin{array}{l}312 \\
(19)\end{array}$ & $\begin{array}{l}224 \\
(34)\end{array}$ & & & & \\
\hline $\begin{array}{l}\text { Balistidae } \\
\text { (n) }\end{array}$ & $\begin{array}{r}1304 \\
(54)\end{array}$ & $\begin{array}{l}51 \cdots \\
(36)\end{array}$ & $\begin{array}{r}2073 \\
(36)\end{array}$ & $\begin{array}{l}2186 \mathrm{~ns} \\
(34)\end{array}$ & 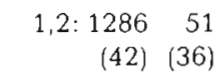 & $\begin{array}{r}2515 \\
(29)\end{array}$ & $\begin{array}{r}2186 \\
(34)\end{array}$ & & & & \\
\hline $\begin{array}{l}\text { Tetraodonti- } \\
\text { formes (n) }\end{array}$ & $\begin{array}{r}36 \\
(55)\end{array}$ & $\begin{array}{l}110 \mathrm{~ns} \\
(36)\end{array}$ & $\begin{array}{r}58 \\
(36)\end{array}$ & $\begin{array}{l}299^{\circ} \\
(34)\end{array}$ & $\begin{array}{rr}2,3: 38 & 110 \\
(15) & (36)\end{array}$ & $\begin{array}{r}56 \\
(19)\end{array}$ & $\begin{array}{l}299 \\
(34)\end{array}$ & & & & \\
\hline Total & $\begin{array}{r}2885 \\
(54)\end{array}$ & $\begin{array}{l}514^{\cdots} \\
(36)\end{array}$ & $\begin{array}{r}5092 \\
(36)\end{array}$ & $\begin{array}{l}5022 \mathrm{~ns} \\
(34)\end{array}$ & $\begin{array}{rr}1-3: 2771 & 514 \\
(47) & (36)\end{array}$ & $\begin{array}{r}5285 \\
(32)\end{array}$ & $\begin{array}{r}5022 \\
(34)\end{array}$ & & & & \\
\hline
\end{tabular}



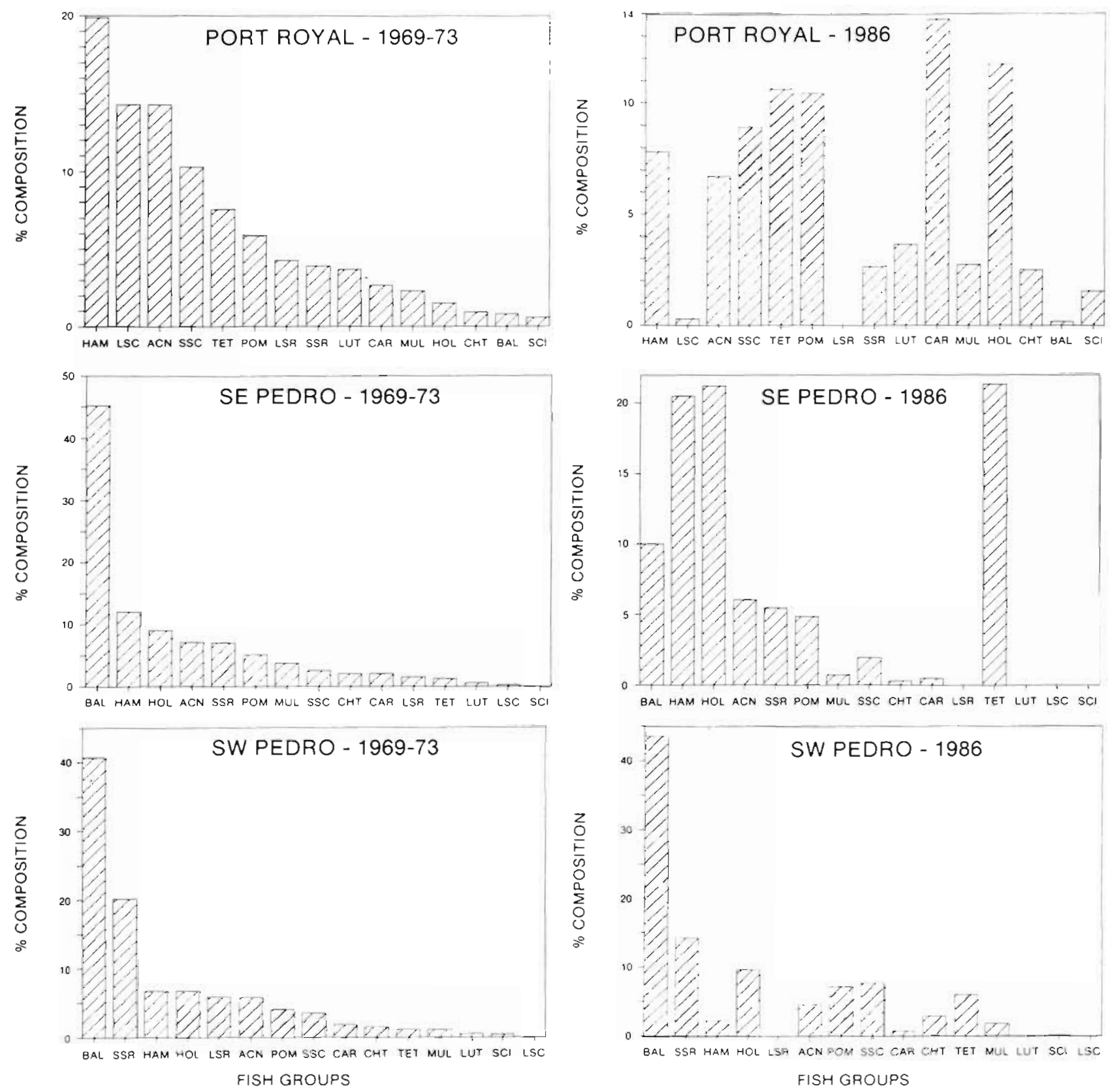

Fig. 4. Percentage composition of reef fish communities around the Port Royal Cays (PRC) and on SE and SW Pedro Bank based upon 1969-73 and 1986 trap surveys (data from Table 3). The composition of the fish communities at PRC and on SE Pedro Bank is significantly different between the 2 surveys (KS test, $p<0.05$ ). Abbreviations for fish groups as in Fig. 2

ers and snappers), secondary commercial groups (smaller serranids and haemulids), and large parrot fishes, and an increase in tetraodontiformes. This may indicate that fishing affects the species composition of an exploited fish community at relatively low exploitation rates or that fishing on the SW Pedro Bank is closer to maximal sustainable levels than available data lead us to believe.

On the other hand, SW Pedro Bank probably only serves in a limited sense as a control area. The Carib- bean Current flows westward over Pedro Bank, so there is likely to be a considerable drift of fish eggs and larvae in that direction. The depletion of groupers, snappers, and large scarids may be due to 'recruitment overfishing' (i.e. reduced reproductive potential) of these species. Although this was caused by overfishing of the 'upstream' portion of the stocks, their recruitment over the entire bank may now be affected. The depletion of other fish groups, which occurred over SE Pedro Bank alone (Fig, 3) may signify more localized 'growth 
overfishing' that has not affected the ability of these species to colonize the bank overall. In general the most abundant fish groups - and presumably the most productive - tended to show relatively little change over lightly-exploited SW Pedro Bank. Thus, when the overall species composition of the community is examined (Fig. 4), only the heavily exploited areas (SE Pedro Bank and PRC) appeared to have undergone a complete re-ordering of the community composition in the interval between the 2 surveys.

Conventional methods of population analysis, which require determination of age-structure and hence of rates of fishing and natural mortality, have generally had little success in tropical reef fisheries (Stevenson 1978, Nicholson \& Hartsuijker 1982, Munro 1983). Age determination is often not yet feasible, and attempts to use length-frequency methods are confounded by sizeselectivity of the gear, migrations at different life-history stages, and lack of well-defined cohorts. The changes in community composition that we observed, which are presumably based upon differences in lifehistory traits (e.g. age at maturity) and catchability, could provide useful indices for fishery management if their relationship with fishing effort were quantified.

The change noted in the heavily exploited areas, which involved the re-ordering of dominant fish groups and the virtual disappearance of several others, was not initially expected. The traps generally in use are a simple passive gear that saturate at relatively low levels of catch. In temperate and boreal marine systems, the dominant fish groups (e.g. clupeoids, gadoids, pleuronectiformes) generally withstood centuries of fishing activity and only showed signs of overfishing relatively recently, following the introduction of powerful and sophisticated fishing technologies. At first glance, this contrast between high and low latitude fish communities would seem to support May's (1976) hypothesis, developed from modelling studies, that complex ecosystems, such as reefs, are more sensitive to perturbations, such as fishing, than simpler ones.

However, several factors lead us to approach with caution simple comparisons of reef and higher latitude fisheries. The area of reef forms a narrow zone around most Caribbean states, and most of these fish groups are not only restricted to the reef, but have small home ranges within the reef system. The reef area on Pedro Bank is largely limited to the east and the southern edge of the bank (Dolan 1972). There are no seasonal fishing closures, and areas around fishing bases (e.g. PRC and SE Pedro Bank) continue to be fished after the larger, slower maturing and more valuable species have been depleted. There is a market for the remaining species, a lack of alternative economic opportunity, and significant cost and difficulty associated with moving the traps and tending them in more remote areas.
Traps may also be more effective than they initially appear, given the relatively fixed nature of many reef fish populations. There is evidence that catch rates for many species are locally depressed after only the second or third set in the same location (Nicholson \& Hartsuijker 1982). Thus, although actual rates of fishing mortality are not available, it seems that artisanal reef fishermen are able to concentrate their effort upon and deplete local stocks to a far greater extent than fishermen in the pre-modern period in higher latitudes, where the fish range over relatively large coastal systems.

The pattern of change noted in these reef systems is virtually opposite to that observed in the Gulf of Thailand, a well-studied tropical demersal trawl fishery. Pauly (1979) noted that small prey species declined most rapidly in the Gulf of Thailand following the onset of intensive trawl fishing, and larger predators, such as the lutjanids, were less affected. Pauly hypothesized that prey species in tropical ecosystems are exploited by their natural predators near the point of their maximal sustainable yield in the pre-fishery period, rendering them more sensitive to additional, fishery-induced mortality. This hypothesis is not supported by evidence from the Port Royal and Pedro Bank reef fisheries, where the larger fish generally declined most substantially. Other factors which may contribute to the apparent differences between the 2 fisheries include greater selectivity of traps than trawls for the larger piscivores and the concentration of the trawl fishery in nearshore areas of shrimp concentration, which provided the larger predators with a partial refuge offshore. The territorial behavior of reef fish, combined with their limited habitat and depth preferences, renders them particularly vulnerable to depletion.

Further study is required to determine possible secondary effects of the decline of major piscivorous (serranid and lutjanid) and herbivorous (acanthurid and large scarid) fishes. Are small untrapped fish groups, such as labrids and pomacentrids, increasing due to reduced competition and predation? To what extent does the reduction in large herbivorous scarids, as opposed to increased nutrient loads, contribute to the increased algal cover noted on reefs around PRC (H. Witter, Botany Dept., Univ. of West Indies, Kingston, Jamaica, unpubl.) These uncertainties, combined with the rapid depletion of primary (large serranids and lutjanids) and secondary (haemulids) commercial groups and the increasing predominance of commercially less-desirable groups (holocentrids and tetraodontiformes), highlight the need for fishery management.

Acknowledgements. This work was supported by a grant to R.W. from the Office of Undersea Research, NOAA and to J.A.K. from Operating and International Collaborative 
Research grants from NSERC and a sabbatical leave grant from Dalhousie University. P. Reeson kindly provided the data from the 1969-73 survey, and A. Larson critically reviewed the manuscript. The cooperation of I. Goodbody, the staff of the Port Royal Marine Laboratory, and captain and crew of the 'Undersea Hunter' are gratefully acknowledged.

\section{LITERATURE CITED}

Caribbean Fishery Management Council (1985). Fishery management plan, final environmental impact statement, and draft regulatory impact review, for the shallow-water reeffish fishery of Puerto Rico and the U. S. Virgin Islands. Hato Rey, Puerto Rico

Dolan, P. (1972). Genesis and distribution of recent sediments on Pedro Bank. Ph. D. thesis, University College, London

Gosse, P. H. (1851). A naturalist's sojourn in Jamaica. In: Stewart, D. B. (ed.) Gosse's Jamaica 1844-45. Institute of Jamaica Publications, Kingston, p. 4. 39-41

Hay, M. E. (1984). Patterns of fish and urchin grazing on Caribbean coral reefs: are previous results typical? Ecology $65: 446-454$

Legendre, L., Legendre, P. (1983). Numerical ecology. Elsevier, New York

May, R. M. (1976). Patterns in multi-species communities. In: May, R. M. (ed.) Theoretical ecology. W. B. Saunders Co., Philadelphia, p. $142-162$
Munro, J. L. (ed.) (1983). Caribbean coral reef fishery resources. Int. Center for Living Aquatic Resources Management, Manila

Munro, J. L., Thompson, R. (1983). Areas investigated, objectives and methodology. In: Munro, J L. (ed.) Caribbean coral reef fishery resources. Int. Center for Living Aquatic Resources Management, Manila, p. 15-25

Neudecker, S. (1979). Effects of grazing and browsing fishes on the zonation of corals in Guam. Ecology 60: 666-672

Nicholson, W. Hartsuijker, L. (1982). The state of the fisheries resources of the Pedro Bank and south Jamaica shelf. FAO Fish. Rep. 278 (Suppl.): 215-254

Norusis, M. J. (1986). SPSS/PC+: SPSS for the IBM PC/XT/ AT. SPSS, Inc., Chicago

Pauly, D. (1979). Theory and management of tropical multispecies fish stocks; a review, with emphasis on the southeast Asian demersal fisheries. Int. Center for Living Resources Management, Manila

Sahney, A. K. (1982). Sample survey of the fishing industry in Jamaica - 1981. Min. of Agriculture, Kingston, Jamaica

Sokal, R. R., Rohlf, F. J. (1981), Biometry, 2nd edn. W. H. Freeman and Co., New York

Stevenson, D. K. (1978). Management of a tropical fish pot fishery for maximum sustainable yield. Proc. Gulf Carib. Fish. Inst. 30: 95-115

Thompson, R., Munro, J. L. (1983). The biology, ecology and bionomics of the Jacks, Carangidae. In: Munro, J. L. (ed.) Caribbean coral reef fishery resources. Int. Center for Living Aquatic Resources Management, Manila, p. 82-93

This article was presented by Professor P. J. Wangersky; it was accepted for printing on January 22, 1988 